\title{
Complicated Pneumococcal Meningitis: A Diagnostic and Therapeutic Challenge
}

\author{
Sura Ahmed Al-Doory ${ }^{a}$ Alia Magzoub ${ }^{a}$ Nikhil Pawar ${ }^{b}$ Mahmoud Radaideh ${ }^{a}$ \\ Shafeeka Mohammed Saleh ${ }^{b}$ Mohammed Ali Al Sabbah ${ }^{b}$ Fatima Mir ${ }^{\mathrm{c}}$ \\ aPediatric Hematology Department, Latifa Women and Children Hospital, Dubai, United Arab Emirates; ${ }^{\text {bPediatric }}$ \\ Neurology Department, Latifa Women and Children Hospital, Dubai, United Arab Emirates; 'Pediatrics Department, \\ Latifa Women and Children Hospital, Dubai, United Arab Emirates
}

\section{Keywords}

Pneumococcal meningitis · Vasculitis · Corticosteroids

\begin{abstract}
Meningitis is an uncommon complication of head trauma. Vasculitis in bacterial meningitis is seen in $9-25 \%$ of adults while neurological deficits in bacterial meningitis are seen in about one-third of children. We report a 5-year-old boy, previously healthy who was admitted in March 2019 to Latifa Women's and Children's Hospital, Dubai, UAE, with pneumococcal meningitis. One day before presentation, he had a history of fall with head trauma while running at school. Initial brain CT scan was normal. Few hours after admission, the child was noticed to be drowsy with cold extremities and mottled skin. He was shifted to PICU and, ultimately, he required intubation and mechanical ventilation. The child continued spiking high-grade fever with deterioration in the neurologic status. His GCS deteriorated to 4/15 with decerebrations posture. He underwent serial brain imaging which revealed multiple chronic infarcts with hydrocephalic changes due to ongoing cerebral vasculitis. The child was started on steroid therapy on 28 April 2019 after which his condition improved at an incredible pace.

(c) 2021 The Author(s).

Published by S. Karger AG, Basel
\end{abstract}

\section{Introduction}

Vasculitis in bacterial meningitis is seen in $9-25 \%$ of adults, while neurological deficits are seen in about onethird of children. Streptococcus pneumoniae meningitis may cause neurological injury by various mechanisms including meningeal inflammation, cerebral edema, necrosis, and intracranial hemorrhage. Additionally, there is evidence that cerebral infarction postbacterial meningitis may be secondary to vasculitis [1]. Vergouwen et al. [2] hypothesized that diffuse cerebral intravascular coagulation is an additional explanation for cerebral infarction in patients with pneumococcal meningitis. Meningitis is an uncommon complication of head trauma, and posttraumatic meningitis is a rare complication of head injury in children and can occur with no sign of dural tear or basal skull fracture [3]. Pneumococcal meningitis, often recurrent, have been reported in patients with histories of prior head trauma. Unless a CSF leak is present, there is no routine recommendation for pneumococcal vaccination of persons with a history of head injury or brain surgery [4].
(C) 2021 The Author(s).

Published by S. Karger AG, Basel

This is an Open Access article licensed under the Creative Commons Attribution-NonCommercial-4.0 International License (CC BY-NC) (http://www.karger.com/Services/OpenAccessLicense), applicable to the online version of the article only. Usage and distribution for commercial purposes requires written permission.
Correspondence to:

Sura Ahmed Al-Doory, saaldoory@ dha.gov.ae 

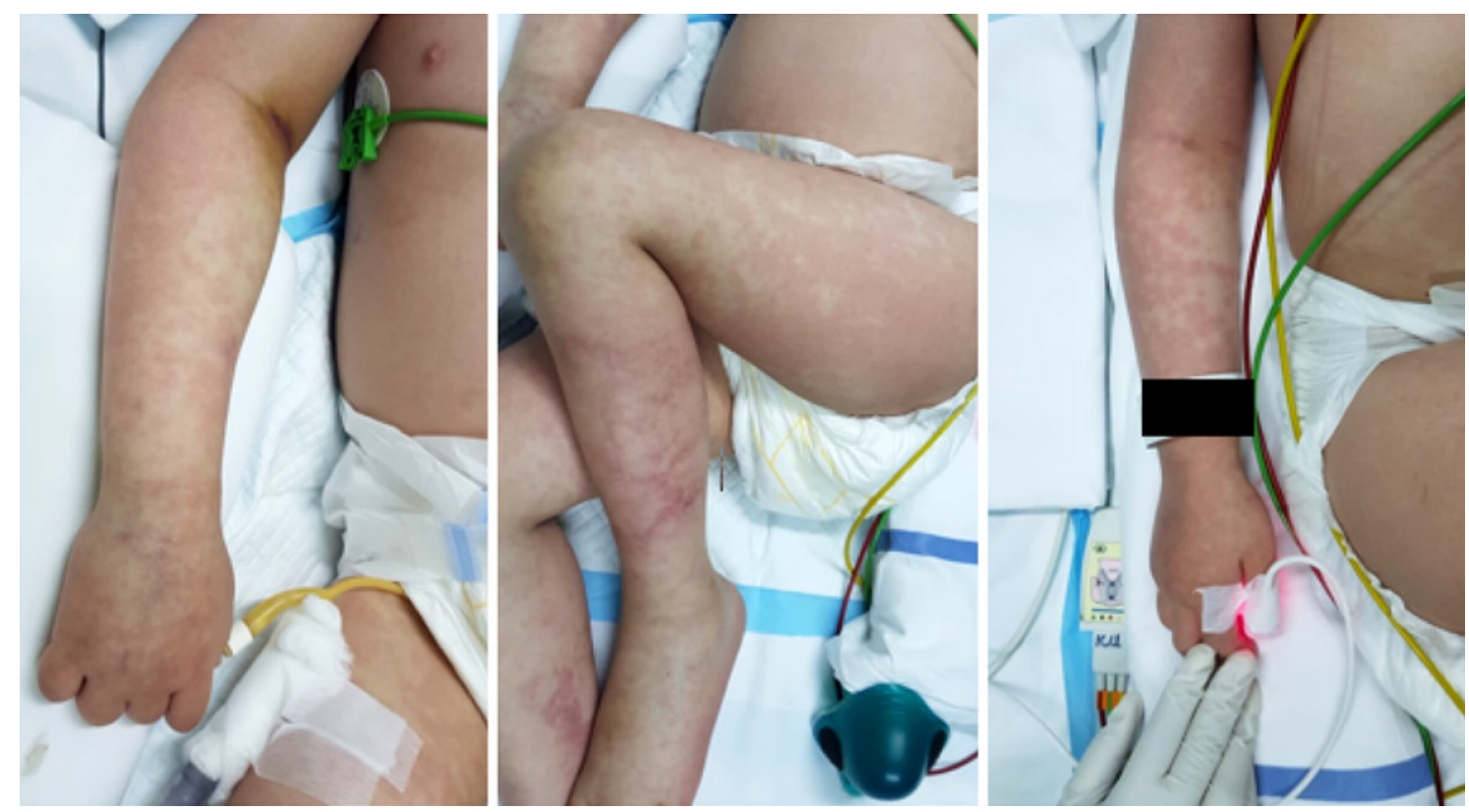

Fig. 1. Generalized lacy, well-defined erythematous, and pruritic rash flaring up during febrile episodes only.

\section{Case Report}

The patient is a 5-year-old previously healthy and fully vaccinated male. He presented to the ED with 2 days' history of fever and headache, followed by 1 day of increasing somnolence and poor oral tolerance. Initial interviewing revealed head trauma at school, sustained prior to onset of headache followed by fever upon returning home. There was no loss of consciousness, seizure activity, upper respiratory tract symptoms, and recent sick contact/travel abroad. Clinical examination was remarkable for a febrile, alert, and oriented but irritable child with neck stiffness.

Emergent CT brain to rule out intracranial bleed was arranged for, and blood work-up was requested as well. The former suspicion was ruled out with normal CT brain features, and with initial laboratory reports suggestive of an underlying infection, the diagnosis of acute bacterial meningitis was prioritized. IV Ceftriaxone was initiated, and lumbar puncture was performed promptly thereafter. Preliminary spinal fluid analysis revealed Gram-positive cocci along with predominant neutrophilia and low glucose/ raised protein. Hence, IV Vancomycin was added onto to therapy.

Clinical condition deteriorated in the forthcoming hours, with features of septic shock requiring transfer to the intensive care unit. A diffuse petechial rash erupted over limbs and child's neurologic status deteriorated requiring elective intubation. IV immunoglobulin was administered once, and phenobarbitone was started for neuroprotection.

Within $18 \mathrm{~h}$ after admission, blood and spinal fluid cultures were reported with growth of S. pneumoniae resistant to Ceftriaxone. Antibiotic therapy was adjusted to vancomycin and meropenem to suit culture sensitivity report.

Sadly, the treatment over the coming few days failed to resolve high-grade fever, rash, and poor neurological status. Occult sources of infection were sought with repeat blood/urine/respiratory cultures, respiratory viral screening, and abdominal ultrasound. Yield was only of underlying enterovirus and rhinovirus on nasal PCR, with FBC/CRP, and all other reports with improving parameters. Repeat CT brain images revealed features of cerebritis and minimal frontal subdural effusion. Septrin was then added to care in view of culture sensitivity and grim clinical circumstances.

Two weeks into admission, the child had only strengthened to allow extubation to room air. He remained, however, in a neurologically miserable condition, lying aloof with fixed spastic flexion of upper and lower limbs, poor intermittent visual fixation, occasional weak purposeless moaning, and failure to respond to voice or touch. Fever pattern was high grade and relentless, with no period of defervesence since admission.

On Day 12, MRI brain revealed meningoencephalitis with early empyema formation along the frontal convexity on either side and features suggestive of Wernicke's encephalopathy. Care was upgraded with thiamine and rifampicin after multidisciplinary consultation. Drainage of subdural fluid collection was avoided in view of benign radiological features and weak clinical indications.

Thiamine and the arsenal of antibiotics all failed to improve the child's condition; hence, lumbar puncture was repeated along with septic screening parameters. Virology analysis as well as culture of spinal fluid was negative, and all blood work-up paradoxically showed consistent improvement. Immune status screening as well as screening for autoimmunity both yielded nil.

Super-added to fever and neurologic debility, the child then developed bilateral hip and knee joint swelling along with a diffuse fine erythematous lacy rash over entire body (Fig. 1). Joint ultrasonography pointed to simple effusions, rash persisted despite regular antihistamines, and notably demonstrated peaks with fever.

The newly manifested combination of fever, rash, joint swelling, and neurologic debility consolidated the fact that the child was suffering from much more than an isolated neurological infection. 


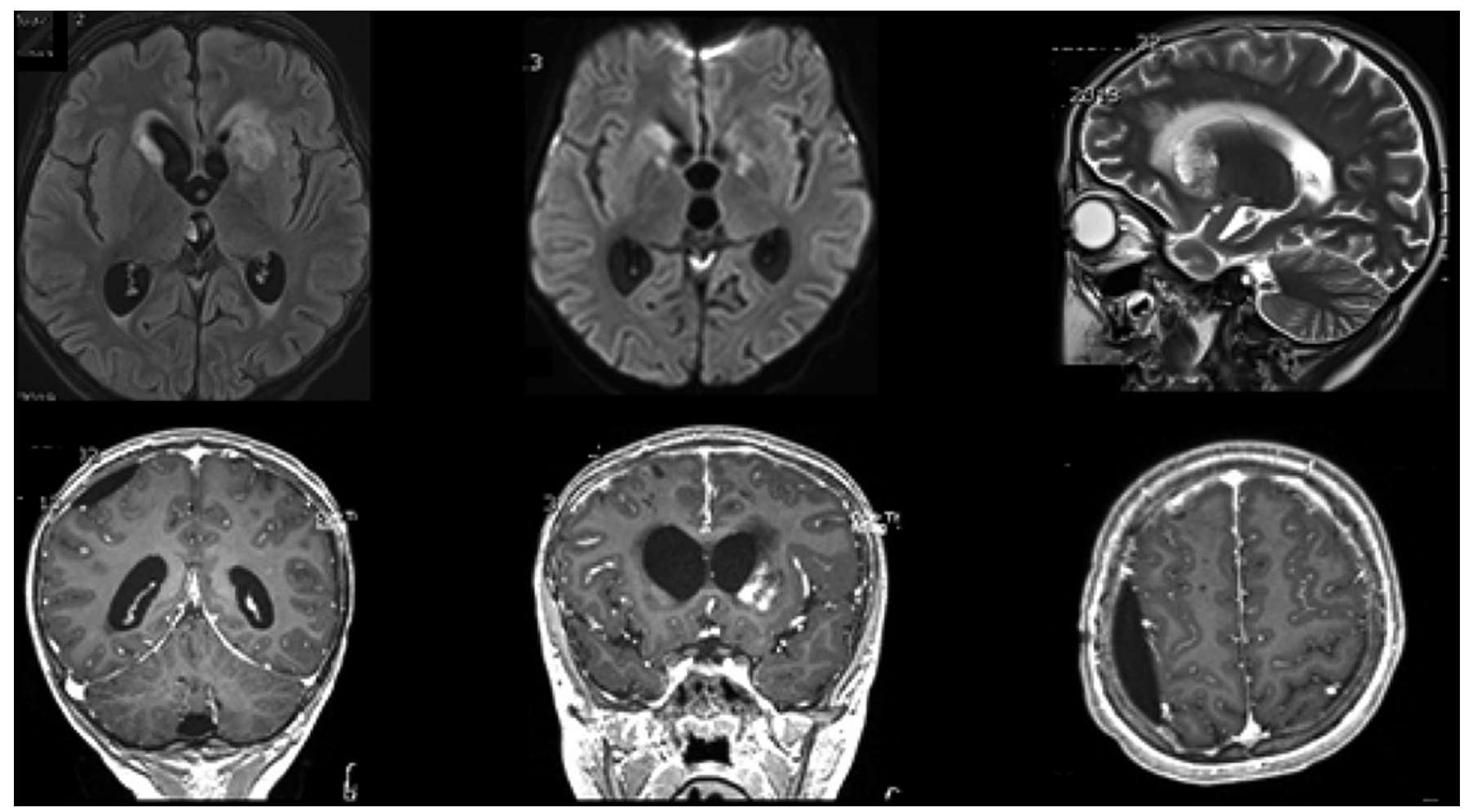

Fig. 2. Multiple acute/subacute infarcts noted in both basal ganglia and anterior limb of internal capsule could be sequelae of vasculitis secondary to meningitis. Mild to moderate hydrocephalic changes with minimal periventricular CSF seepage.

Repeat MRI brain diffusion weighted images revealed multiple acute and subacute cerebral infarctions (Fig. 2).

A consolidating diagnosis of systemic vasculitis secondary to $S$. pneumoniae meningitis was then established. Methylprednisolone therapy followed by oral steroids was initiated, which brightened the child's condition remarkably. Within first day of steroid therapy, fever, rash, and all lab anomalies resolved. Two weeks down the line, the child was able to rest comfortably with all limbs extended, move all limbs independently with near full power, mobilize with minimal support, speak in both English and native language with excellent social skills, feed and toilet self, and read and write as per his developmental level prior to illness. Final MRI brain for follow-up demonstrated minimal interval changes, and it was decided to repeat brain imaging in event of clinical deterioration.

Overall, a total of 3 days of ceftriaxone, 6 days of Septrin, 10 days of rifampicin/thiamine, 5 and 7 weeks of vancomycin and meropenem, respectively, and 19 days of steroids were completed during inpatient stay, with the patient discharged home on a 2-month-long tapering course of steroid therapy.

Follow-up 4 months postdischarge revealed an active, healthy, and thriving child with no neurologic deficits or seizure activity and a bilaterally passed hearing test. He was discharged from follow-up and remains well with no regular medications or residual sequalae of systemic vasculitis.

\section{Discussion}

Acute bacterial meningitis is usually fatal without treatment, and prompt diagnosis coupled with timely administration of appropriate parenteral antibiotics is essential to save lives. The 3 vaccine-preventable organisms are Haemophilus influenzae type b, S. pneumoniae, and Neisseria meningitidis [5].

S. pneumoniae infection is a leading cause of pneumonia, meningitis, and septicemia worldwide, and results in approximately 1 million deaths in children under the age of 5 years annually $[6,7]$. Certain risk factors such as cochlear implants, skull fractures, and complement system deficiency have been associated with both recurrent and severe pneumococcal meningitis $[3,4]$. Overall, vaccination with PCV-13 has led to a decline in infection rates; however, both antimicrobial-resistant and nonvaccine serotypes are proving themselves to be a growing threat [8-12].

Direct tissue damage via bacterial invasion and the consequent host hyperinflammatory response play a partnered role in the deleterious neurologic disease features [13]. Parenchymal damage may be secondary to the 
increased intracranial pressure, cytotoxic and vasogenic edema, herniation, local leukocyte infiltration or abscess formation, as well as by cortical necrosis and hippocampal neuronal loss $[9,14,15]$.

A profound understanding of the underlying pathophysiology of pneumococcal meningitis can be obtained via histopathologic study of cerebral tissue. In 2016, JooYeon Engelen-Lee et al. published an invaluable report of autopsy findings in 31 brains with proven pneumococcal meningitis. Histopathologic discoveries included inflammation of medium-large arteries in 30 brains (97\%), obstruction of vascular lumen in 14 (of 31 cases, $45 \%$ ), cerebral hemorrhage in $24(77 \%)$, cerebritis in $24(77 \%)$, thrombosis in $21(68 \%)$, infarction in $19(61 \%)$, and ventriculitis in 19 (of 28 cases, 68\%). Analysis of vascular involvement in particular was notable for significant inflammatory and occlusive changes occurring both in the early and late phases of disease (defined as within or beyond 7 days of developing meningitis, respectively). To sum up, the findings strongly suggest that vascular damage is key in the process of brain damage in pneumococcal meningitis [16].

The cerebral vascular involvement in pneumococcal meningitis has been modestly reported both in adults and children, with disease features ranging from hearing loss, hydrocephalus, parenchymal infarctions, cerebritis, and hemorrhages [17-21]. Systemic involvement in the form of a diffuse skin rash has been reported once previously by Theonimfi Tavladaki et al. They encountered a 2-year-old child with pneumococcal meningitis, followed by immunemediated vasculitic rashes and cerebral diffusion restriction diffusion-weighted MRI [1]. Hence, it is clear in sight that pneumococcal infection has the capacity for systemic vasculitis, as was demonstrated in our presented case as well.

Management guidelines regarding choice of antimicrobial agents are far more well defined than for the usage of corticosteroids. To date, only meningitis secondary to Haemophilus influenzae is considered a strong indication for early steroid administration; however, evidence is mixed regarding its benefit in pneumococcal meningitis. Reports range from early use prophylactically, to later on in disease course once vasculitis has been established via neuroimaging. Our reported case adds to the pool of data supporting evidence of prominent vasculitic and hyperimmune reactions to pneumococcal meningitis displaying a remarkable response to this simple yet vital medication. An atypical skin eruption and joint swellings associated with cerebral vasculitis in a child with pneumococcal meningitis has been described in only few cases, and ours may be the first in the Arab region.

Complicated Pneumococcal Meningitis

\section{Conclusion}

Pneumococcal meningitis may be well complicated by systemic manifestations of immune-mediated vasculitis; hence, systemic antibiotic therapy should be coupled with corticosteroid therapy for better prognosis. Furthermore, vasculitis is a well-documented complication of pneumococcal meningitis; therefore, persistent fever despite culture-sensitive antibiotics should prompt neuroimaging with diffusion-weighted MRI to search for an underlying vasculitic process.

\section{Statement of Ethics}

Ethical approval was not required for this case report in accordance with the Dubai Health Authority Research Committee policies. Written and informed consent was obtained from the patient's parents for publishing the case report including accompanying images.

\section{Conflict of Interest Statement}

The authors have no conflicts of interest to declare.

\section{Funding Sources}

The authors have no funding sources to declare.

\section{Author Contributions}

S.A. wrote the manuscript and obtained the guardian's consent. All remaining co-authors have contributed to the critical review and editing of the report.

\section{References}

1 Tavladaki T, Spanaki AM, Ilia S, Geromarkaki E, Raissaki M, Briassoulis G. Unusual exanthema combined with cerebral vasculitis in pneumococcal meningitis: a case report. J Med Case Rep. 2011 Dec;5(1):410.

2 Vergouwen MD, Schut ES, Troost D, van de Beek D. Diffuse cerebral intravascular coagulation and cerebral infarction in pneumococcal meningitis. Neurocrit Care. 2010 Oct 1; 13(2):217-27.

3 Lau YL, Kenna AP. Post-traumatic meningitis in children. Injury. 1986 Nov 1;17(6):4079.

4 Gallivan M, Murray E, Harriman K. Association of Streptococcus pneumoniae meningitis after head injury or brain surgery: California, 2013-2014. In open forum infectious diseases. Infectious Diseases Society of America; 2015. Vol. 2(Suppl 1); p. 1896. 
5 Gómez-Baena G, Bennett RJ, Martínez-Rodríguez C, Wnęk M, Laing G, Hickey G, et al. Quantitative proteomics of cerebrospinal fluid in paediatric pneumococcal meningitis. Sci Rep. 2017 Aug 1;7(1):7042.

6 Engelen-Lee JY, Brouwer MC, Aronica E, van de Beek D. Pneumococcal meningitis: clinical-pathological correlations (meningenepath). Acta Neuropathol Commun. 2016 Mar 22;4(1):26.

7 O'Brien KL, Wolfson LJ, Watt JP, Henkle E, Deloria-Knoll M, McCall N, et al. Burden of disease caused by Streptococcus pneumoniae in children younger than 5 years: global estimates. Lancet. 2009 Sep 12;374(9693):893902.

8 Mizrahi A, Marvaud JC, Pilmis B, Nguyen Van JC, Couzigou C, Bruel C, et al. Emergence of ceftriaxone resistance during a case of pneumococcal meningitis with fatal evolution. Antimicrob Agents Chemother. 2020 Feb 21;64(3):e01958-1019.

9 Hsu HE, Shutt KA, Moore MR, Beall BW, Bennett NM, Craig AS, et al. Effect of pneumococcal conjugate vaccine on pneumococcal meningitis. N Engl J Med. 2009 Jan 15; 360(3):244-56.
10 Oligbu G, Collins S, Djennad A, Sheppard CL, Fry NK, Andrews NJ, et al. Effect of pneumococcal conjugate vaccines on pneumococcal meningitis, England and Wales, July 1, 2000 June 30, 2016. Emerg Infect Dis. 2019 Sep; 25(9):1708-18.

11 Erdem H, Elaldi N, Öztoprak N, Sengoz G, Ak $\mathrm{O}$, Kaya S, et al. Mortality indicators in pneumococcal meningitis: therapeutic implications. Int J Infect Dis. 2013;19:13-9.

12 Bernson-Leung ME, Lehman LL. Cerebrovascular complications of pediatric pneumococcal meningitis in the PCV13 era. Hosp Pediatr. 2016 Jun;6(6):374-9.

13 Barichello T, Generoso JS, Simões LR, Elias SG, Quevedo J. Role of oxidative stress in the pathophysiology of pneumococcal meningitis. Oxid Med Cell Longev. 2013;2013: 371465-7.

14 Darling KE, Niederhauser J, Bervini D, Giulieri S, Daniel RT, Bille J, et al. Cerebral vasculitis complicating postoperative meningitis: the role of steroids revisited. Swiss Med Wkly. 2012;142:w13697.

15 Mook-Kanamori BB, Geldhoff M, van der Poll T, van de Beek D. Pathogenesis and Pathophysiology of Pneumococcal Meningitis. Clin Microbiol Rev. 2011 Jul 6;24(3):557-91.
16 Engelen-Lee JY, Brouwer MC, Aronica E, van de Beek D. Pneumococcal meningitis: Clinical-pathological correlations (meningenepath). Acta Neuropathol Commun. 2016 Mar 22;4(1):26.

17 Khedher A, Sma N, Slama D, Fraj N, Hachfi W, Boussarsar M. Cerebral vasculitis complicating pneumococcal meningitis. Eur J Case Rep Intern Med. 2018;5(5):000819.

18 Poil AR, Shaukat A, Kumar D. Pneumococcal meningitis complicated by cerebral vasculitis, abscess, hydrocephalus, and hearing loss. Case Rep Infect Dis. 2018 Oct 16;2018:1-6.

19 McInnes A. Correspondence. Cambridge University Press; 2020.

20 Ribeiro S, Domingues V, Faria RM, Mendonça T. Invasive pneumococcal disease complicated by cerebral vasculitis, transient diabetes insipidus and spondylodiscitis. BMJ Case Rep. 2013 Aug 19;2013.

21 Ertner G, Christensen JR, Brandt CT. Pneumococcal meningitis with normal cerebrospinal biochemistry and no pneumococci at microscopy, mimicking a stroke: a case report. J Med Case Rep. 2017 Jun 7;11(1):150. 